\title{
Operation State Prediction in Wind Power Integrated Systems Based on Artificial Neural Network
}

\author{
Wang Jiang, Jiping Lu \\ State Key Laboratory of Power Transmission Equipment \& System, \\ Security and New Technology, College of Electrical Engineering, \\ Chongqing University, \\ Chongqing, China
}

\begin{abstract}
With the capacity of integrated wind farm increasing, the reliability issues of power systems could not be ignored. This paper proposes an evaluation method for power system operation state based on elastic back-propagation neural network through the data of the phasor measurement unit. The effectiveness of the proposed method is verified by the IEEE 14-bus system, it has overcome the slow convergence rate problem and the prediction accuracy is acceptable. Condition assessment of power systems operation state is an important approach to improving the operation reliability of power systems.
\end{abstract}

Keywords-wind power; neural network; operation state prediction; elastic back-propagation algorithm; PMU

\section{INTRODUCTION}

With the continuous development of wind power, the proportion of integrated wind power capacity is increasingly growing. Many large-scale wind farms have already been integrated in the power networks. In many countries, the average wind power penetration is $20 \%$ or higher [1]. Till 2010, England has already installed a large amount of renewable energy generation, which is up to $10 \mathrm{GW}$ and the wind power takes up nearly $60 \%$ [2].

As the increasing penetration of wind power, the impact on operation reliability of power system becomes more significant. The influence on grid stability increases due to the randomness and fluctuation of wind power. Under high penetration conditions, a massive wind farms disconnection can lead power system to collapse [3]. At present, variety evaluation methods for power system operation state have been proposed. The probabilistic state evaluation model is built by the Monte Carlo method; the operating states can be analyzed by the state criterion which is made according to load importance and accepted security in Ref. [4]. An operation condition evaluation model for power transformer, which can evaluate the state of every grade and gives a comprehensive assessment, is established on the base of the fuzzy neural network [5]. In order to overcome the Monte Carlo's disadvantage of slow convergence rate and the huge computational resources, the steady state probability of power system can be rapidly analyzed by a Markov state transition probability matrix in Ref. [6]. However, there is seldom research about the evaluation state method for wind power integrated system. This paper uses PMU to collect data at crucial nodes, builds the forecasting model by using the elastic back-propagation algorithm. To predict and evaluate the operation states of wind integrated power system based on the trained neural network. The effectiveness of the proposed method is proved by the simulation results by using an actual example for the wind power system.

The paper is organized as follows. In the next section, the phasor measurement unit structure and optimal placement strategy are given. In Section 3, the BP neural network based on the elastic back propagation algorithm is presented. Section 4 describes the detailed program process. Section 5 discusses the simulation results. Finally, Section 6 presents the conclusion.

\section{PHASOR MEASUREMENT UNIT}

PMU is the key distinction between WAMS (Wide Area Measurement System) and SCADA (Supervisory Control And Data Acquisition) system which is based on the remote terminal unit. PMU can utilize the GPS (Global Positioning System) to obtain high precision clock signal at the same time around the world, and its precision can reach up to 1 us. If the rated frequency is $50 \mathrm{~Hz}$, the angle error of each node is only 0.018 , it can meet the various application demands of power systems $[7,8]$.

\section{A. PMU structure}

PMU can be divided into six modules according to the function [9], shown in Figure 1. Power module provides power supply for each module. Through extensive transformation processes, the A/D sampling module can acquisition analog signals of voltage, frequency, current, etc. Central processing module calculates the corresponding synchronous phasor with the data provided by the A/D sampling module. GPS module is the standard time source of data acquisition and phasor calculation, it will send the sampling pulse signal based on Pulse per second and universal time coordinated respectively into the $A / D$ sampling module and the central processing module. Communication module is connected to the phasor data concentrator or control center. Man-machine interface module includes keyboard, display, etc. 


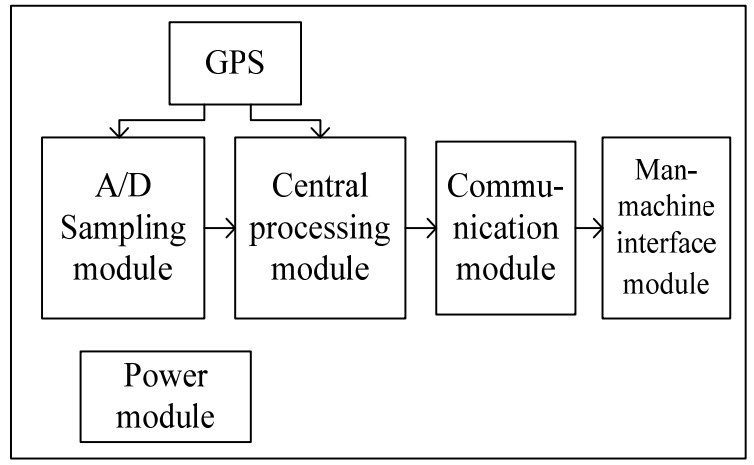

Figure 1. The structure of PMU function modules.

\section{B. PMU placement scheme}

Optimal PMU placement problem mainly focuses on how to use the minimum number of PMU to make sure the observability requirements of system with a big redundancy of data. The optimal strategy can be defined as:

$$
\left\{\begin{array}{l}
\min \{\max R(n, S(n))\} \\
\text { s.t. } \quad U=0
\end{array}\right.
$$

Where $n$ is the number of PMU, $S(n)$ is the location set of PMU, $R$ is the measurement redundancy of system, $U$ is the number of unobservable bus.

For a power system with $\mathrm{n}$ nodes, the power flow equations can be solved by the sparse structure of system. Firstly, the minimum dimension node is taken as initial configuration node. As a starting point, to solve the node power equation with the least unknown node voltage phasor in order. If the equations cannot obtain the new voltage of unknown node, a new PMU should be placed in the node with least number of unknown node voltages $[10,11]$.

\section{BP NEURAL NETWORK}

\section{A. BP neural network modeling}

Artificial neural network is a massive information processing system which consists of a large amount of parallel processing units. ANN can extract rules from the data and present the stable network in the form of weights, obtain the required data by the associative memory and generalization ability. At present, the Back-propagation neural network is used widely because of its strong current processing capability, fault-tolerance ability and capability to quickly and effectively deal with nonlinear system.

BP network is a kind of feed-forward neural network, including the input layer, hidden layer and output layer [12]. In this paper, a three-layered BP network is presented to construct the predict model. The neuron node number of input layer is So, $i=1,2, \ldots, S 0$; the number of hidden layer $\mathrm{S} 1$, the neuron node number of output layer S2. The neuron activation function is as follows:

$$
\left\{\begin{array}{l}
f(x)=\frac{1}{1+e^{-x}}, \quad x \in(-\infty,+\infty) \\
f(x) \in(0,1) \\
f^{\prime}(x)=f(x)(1-f(x)), \quad x \in(-\infty,+\infty) \\
f^{\prime}(x) \in\left(0, \frac{1}{4}\right]
\end{array}\right.
$$

\section{B. Elastic back propagation algorithm}

The conventional BP neural network is easily affected by the initial values of network structure, it will slow down the convergent rate and fall into local minima and saturation region of the activation function. The improved elastic back propagation algorithm is utilized to accelerate the convergence velocity and solve the saturation problem.

The updating value of each weight $\Delta_{i j}{ }^{(t)}$ can be independently determined by the elastic back propagation algorithm. According to the error function $E$, the local gradient value could adaptive learning with the rule as follows [13]:

$$
\left.\Delta_{i j}{ }^{t}\right)=\left\{\begin{array}{cc}
\eta^{+} \times \Delta_{i j}{ }^{(t-1)} & \left(\frac{\partial E^{(t-1)}}{\partial w_{i j}} \times \frac{\partial E^{(t)}}{\partial w_{i j}}>0\right) \\
\eta^{+} \times \Delta_{i j}{ }^{(t-1)} & \left(\frac{\partial E^{(t-1)}}{\partial w_{i j}} \times \frac{\partial E^{(t)}}{\partial w_{i j}}<0\right) \\
\Delta_{i j}{ }^{(t-1)} \quad(\text { eles })
\end{array}\right.
$$

In this formula, $t$ stands for the training times, $E$ is the square sum of error, $\frac{\partial E^{(t)}}{\partial w_{i j}}$ is the summing gradient data of all learning modes, $W$ is the weight vector.

Considering the balance between relative error and absolute error, the error function is expressed as:

$$
E=\frac{1}{4}\left\{A \sum_{k=1}^{s 2}\left(1-\frac{y 2_{k}}{d_{k}}\right)^{2}+\sum_{k=1}^{s 2}\left(d_{k}-y 2_{k}\right)^{2}\right\}
$$

Where $A$ is the expected output of the whole sampling data, $y$ is the actual output and $d$ stands for the expected output.

\section{PROgRAM PROCESS}

The training of the $\mathrm{BP}$ neural network includes the following steps:

(i) Acquire necessary information from the PMU at key nodes;

(ii) Input the required parameters, the neuron node number of each layer are set. 
(iii) Initializations for weights and thresholds, the gradient of $W, b$ are set to be zero matrixes.

(iv) To determine whether it meets the defined goals, if $M E<\varepsilon$, stop the learning cycle and expert the outputs. Otherwise, adjust $W$ and $b$ for next time training and update the weights and thresholds.

(v) The loop will stop, if either of those conditions is fulfilled: the training time $t>M A X_{-} N$ or $M E<\varepsilon$.

(vi) If $M E<\varepsilon$, the trained neural network is successful with acceptable forecasting error. The weights and threshold value matrixes can be exported as outputs.

The prediction results can be calculated by the trained neural network with the weights and threshold value matrixes $W, b$.

\section{CAse STUdy}

In order to validate the effectiveness of the proposed method, the IEEE-14 bus system is considered as shown in Figure 2. The wind farm is connected to the system by a $110 \mathrm{kV}$ line at node 14 , which is consist of 30 fixed speed wind turbines. Each of the wind turbine is of $2 \mathrm{MW}$ capacity. To study the effect of wind power penetration on the power system stability, it is assumed that loads of all nodes remain constant. This study used the active power and reactive power as the training input vector variables and selected the frequency and voltage as the output vector variables which can be used to assess the security state of the integrated system. The PMU is placed according to the principle of optimal allocation which is discussed before. Table I shows the detailed learning parameters of the training network.

The frequency of wind power integrated system mainly influenced by the random output power of wind farm, without consideration for load fluctuation. The intermittent and random fluctuation of wind speed can cause tremendous stress on the stability of power system frequency, which will bring fluctuation of the wind farm output. Figure 3 describes the frequency response of integrated power system. The system frequency increases when the output power of wind farm increases, when the wind is weak, the frequency decreases with the decrease of output power of wind farm. The trained BP neural network can accurately reflect actual situation of frequency, the predict frequency curve can track the variation trend of target value in time.

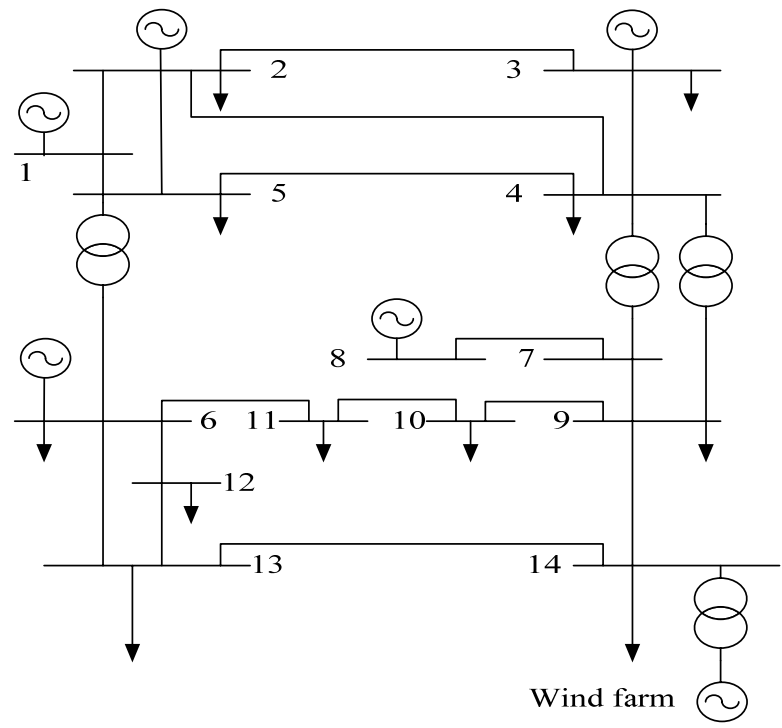

Figure 2. IEEE-14 bus system integrated with wind farm.

TABLE I. LEARNING PARAMETERS.

\begin{tabular}{|c|c|}
\hline Learning Parameters & Value \\
\hline Terminal goal error $\boldsymbol{\varepsilon}$ & 0.001 \\
\hline Maximum iterations MAX_N & 10000 \\
\hline Initial value of weight update $\Delta_{0}$ & 0.1 \\
\hline Maximum value of weight update $\Delta_{\max }$ & 1.2 \\
\hline The increscent multiple of the value of weight update $\eta^{+}$ & \\
\hline The decrescent multiple of the value of weight update $\eta^{-}$ & 0.5 \\
\hline
\end{tabular}

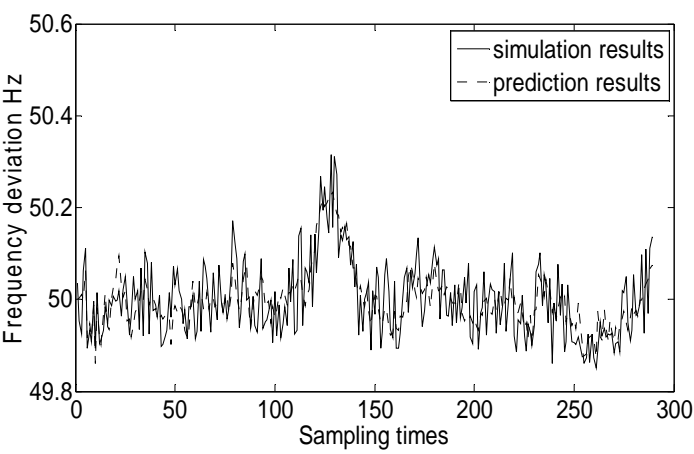

Figure 3. Frequency deviation of wind power integrated system. 


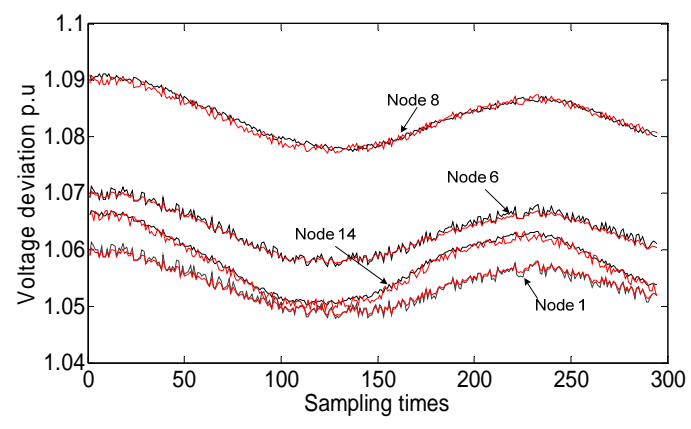

Figure 4. Voltage deviation of wind power integrated system.

TABLE II. ANALYSIS ON FORECAST RESULTS ERRORS.

\begin{tabular}{|c|c|c|}
\hline Forecast Result & MAE & RMSE \\
\hline Frequency & 0.0491 & $5.93 \%$ \\
\hline Node 1 & 0.0377 & $4.7162 \%$ \\
\hline Node 6 & 0.0429 & $5.1975 \%$ \\
\hline Node 8 & 0.0719 & $8.6496 \%$ \\
\hline Node 14 & 0.0647 & $7.9392 \%$ \\
\hline
\end{tabular}

Figure 4 shows the voltage curves of key nodes. The black curves stand for the actual value of voltage and the red curves are the forecasting curve. In this paper, all wind turbines are squirrel-cage asynchronous motor which will absorb the reactive power from grid. The compensating capacitor is connected to the system to prevent voltage collapse. As shown in Figure 4, the voltage of integrated power system would be no significant downturn with the help of compensating capacitor. When the wind velocity increases, more wind turbines operate in parallel to the grid. Although the active power of wind farm increases, more reactive power is absorbed from the grid side which leads to a decline in voltage. On the other hand, when the wind turbines are cut off, the voltage climbs up once again. From Figure 4, the voltage fluctuations of all nodes are acceptable, the results meet the requirement of voltage stability, the integrated power system can maintain its reliability under this operate condition. In particular, because of the position closer to the wind farm, the voltage volatility of node 14 is more obvious than the other nodes.

The root mean square error (RMSE) and mean absolute error (MAE) corresponding to each forecast result are shown in Table II. RMSEs of each forecast result are significantly less than $10 \%$. The largest RMSE is only $8.6496 \%$, it can indicate that elastic-BP neural network is suitable for evaluating operation state of wind power integrated system, and the prediction neural network utilized in this paper is effective.

\section{CONCLUSIONS}

A new method for forecasting operation states of wind farm integrated power system has been proposed in this paper. The output power fluctuation of wind farm will cause frequency and voltage deviation, which brings a number of challenges to the grid operator. The presented BP neural network can accurately reflect the deviation of frequency and voltage with reliable precision. This method can provide helpful data support for grid operators to evaluate the stability of wind power integrated power systems.

\section{ACKNOWLEDGMENTS}

The research work was supported by the Scientific Research Foundation of stage key lab of power transmission equipment and system security under Grant No. 2007DA10512712205.

\section{REFERENCES}

[1] L. Holdsworth, J. Ekanayake, N. Jenkins, Power system frequency response from fixed speed and doubly fed induction generator-based wind turbines. Wind Energy, 7, pp. 21-35, 2004.

[2] J. Lin, Y.Z. Sun, P. Sørensen, Method for assessing grid frequency deviation due to wind power fluctuation based on "time-frequency transformation.” IEEE Trans. Sustain. Energy, 3(1), pp. 65-73, 2012.

[3] A. Causebrook, D.J. Atkinson, A.G. Jack, Fault ride-through of large wind farms using series dynamic braking resistors. IEEE Trans Power Sys. 22(3), pp. 966-975, 2007.

[4] D. Ming, L. Sheng-hu, W. Hong-bin, et al., Analysis of power system operation state based on adequacy and security. Proceedings of the CSEE 24(4), pp. 43-49, 2004.

[5] L. Ruijin, W. Qian, L. Sijia, et al., Condition assessment model for power transformer in service based on fuzzy synthetic evaluation. Automation of Electric Power Systems, 32(3), pp. 70-75, 2008.

[6] W. Yong, H. Xueshan, D. Ying, Markov chain-based rapid assessment on operational reliability of power grid. Power System Technology, 37(2), pp. 405-410, 2013.

[7] A.G. Phadke, Synchronized phasor measurements in power system. IEEE Computer Applications in Power, 6(2), pp. 10-15, 1993.

[8] J.B. Carvalho, F.M. Barbosa, A modern state estimation in power system energy. International Conference on Electric Power Engineering, Budapest, 1999.

[9] Z. Xin, L. Jiping, W. Yang, et al., Reliability assessment of WAMS based on a combined hardware and software probability model of phasor measurement units. Automation of Electric Power Systems, 33(16), pp. 19-23, 2009.

[10] W. Keying, M. Gang, X. Han, et al., Placement of phasor measurement unit for direct solution of power flow. Proceedings of the CSEE, 19(10), pp. 14-17, 1999.

[11] F.J. Marin, F. Garcia-Lagos, G. Joya, et al., Genetic algorithms for optimal placement of phasor measurement units in electrical networks. IEE Electronics Letters, 39(19), pp. 1403-1405, 2003.

[12] J. Zheng-yuan, T. Li, Short-term power load forecasting based on Fuzzy-RBF neutral network. International Conference on Risk Management \& Engineering Management, pp. 349-352, 2008.

[13] W. Jiang, J. Lu, Frequency estimation in wind farm integrated systems using artificial neural network. International Journal of Electrical Power and Energy Systems, 62, pp. 72-79, 2014. 